\title{
Application of Cooperative Distributed Predictive Control in Power Grid Frequency Adjustment
}

\author{
Hamidreza Iranmanesh ${ }^{1}$, Ahmad Afshar ${ }^{2, *}$ \\ ${ }^{1}$ Department of Electrical Engineering, Amirkabir university of Technology, Tehran, IRAN \\ ${ }^{2}$ Department of Electrical Engineering, Amirkabir university of Technology, Tehran, IRAN \\ "Corresponding author: aafshar@aut.ac.ir
}

\begin{abstract}
One of the efficient control methods is the model predictive control(MPC) that calculates appropriate system inputs to reduce the difference between the desired and the system predicted output. One of the important applications of this strategy is in power grids for load-frequency control and power balancing. Different architectures of MPC are applied in large scale structures such as power grid. In this paper a feasible cooperation based MPC(FC-MPC) controller has been used as a suited solution to control problem with relatively strict constraints. Also it can be used as one of options for control system of smart grids in future. In this paper, a power grid with 4 control zones has been chosen as a case study. The simulation results indicate the superior performance of the discussed control method in comparison with the traditional control of load- frequency.
\end{abstract}

Keywords: frequency control, power balancing, predictive control, smart grid 


\section{INTRODUCTION}

Whit regard to the different types of equipment and also number and orders of subsystems which used in power grids, such grids can be considered as large-scale systems. More detailed study is available in [1]. Centralized control of such systems in terms of the complexity of calculations and the large number of variables is a difficult and impractical task. An appropriate solution is to solve the control problem through decentralized or distributed architecture by separating the overall system into smaller subsystems $[2,3]$.

One of the important problems in power grids is frequency adjustment within an admissible range and balancing between generation and consumption which has an effect on power quality. Currently, one of the most effective control methods is MPC that is appropriate for multivariable constrained systems and industrial applications.

The problem of load-frequency control in power grids can be solved in a good way through predictive control $[4,9,11,12]$. There is a comparison of control methods for controlling the frequency of a power grid in [4]. In this regard, in the present paper, changes in system characteristics and related parameters are used to examine control methods in new conditions more precisely and completely.

The case study is a power grid with 4 control zones each of which has local generation and load. The control method used in this study is introduced in [8]. It can guarantee system stability regarding to the related conditions.

Among the implemented innovations in this paper, is that the load changes are applied to the system in the form of pulse rather than stepwise and with much delay (3 times) in comparison with [4] to examine the system's performance and its speed to return to steady state after successive load changes.
Also, load disturbance is increased up to $40 \%$ and applied to the system in a new situation so that in zone 3 it appears as a load increase and in zone 2 it appears as a load decrease. Moreover, the range of control input changes is decreased by $70 \%$. This limitation prevents the saturation of input signal. Finally, in order to evaluate the overall performance of system, total error is obtained and plotted. It is a weighted combination of frequency deviations and transmission power changes.

Therefore, the stricter conditions and constraints are selected to examine the system's performance more precisely and completely in new situations and good performance of the used control method is shown.

The rest of this paper is organized as follows: Section 2 begins by defining MPC strategy and then explains architectures of this strategy in the large scale systems.

The optimization problem for distributed MPC is given in Section 3.

Section 4 shows a simulation example of AGC and the discussed method applied to a power grid with a number of control zones. Numerical simulations are included to compare the FCMPC approach with traditional method.

The simulation results are discussed in section 5. Finally, this paper is concluded in Section 6.

\section{Model Predictive Control}

In the MPC strategy, a model is used for predicting the future states and outputs of system over a finite horizon called the prediction horizon. Then, regarding the difference between the predicted and desired output; an optimization problem is solved to minimize the related error. The control input is calculated over a finite horizon called the control horizon but only the first step of the calculated input is applied to the system at 
each sampling time. At the next sampling time, this process is repeated by moving the horizon forward. So, this type of control method is termed as receding horizon or moving horizon method. For more information one can refer to [5].

\subsection{Predictive control in large scale structure}

As mentioned in the introduction, due to computational complexities in centralized predictive control method in large-scale systems, in many cases it is not practical. Therefore, decomposition a large scale system into various subsystems produces a more appropriate structure for control system. So that each subsystem has its own local controller. One architecture of this structures is known as decentralized MPC. In this case, it is assumed that no interaction exists between the local controllers. Here, due to lack of some communications among controllers, system performance may be degraded which may lead to stability problems. This type of control can be acceptable for systems with poor communication among their subsystems. Some aspects of this control method have been studied in [6].

Another architecture of this structure is called distributed control. It has neither the computational complexity of a centralized architecture nor the performance degradation of a decentralized architecture. For this reason, a type of this architecture has been used in this paper as described in the next sections.

\subsection{Distributed MPC}

In distributed architecture, the controllers can communicate with each other. A special type of it is known as feasible cooperation based MPC $[8,9]$. In this framework, a global optimization problem is solved cooperatively to calculate each control input. Here, system stability is guaranteed providing that the specific conditions are satisfied.

\section{SYSTEM SPECIFICATIONS}

Consider a system consisting of M subsystems with the overall state space equation given by:

$x(k+1)=A x(k)+B u(k)+B_{d} d(k)$

where $x(k) \in R^{n}$ is the state, $u(k) \in R^{m}$ is the control input and $d(k) \in R^{s}$ is the bounded disturbance at time instant $\mathrm{k}$ and $A \in R^{n \times n}, B \in R^{n \times m}, B_{d} \in R^{n \times s}$.

Distributed optimization problem

The control law for $j$-th $Z_{+}$subsystem is obtained by solving the optimization problem as follows:

$$
\begin{aligned}
& \Phi_{f c_{j}}^{o}=\min _{\left[u_{j}(\mathrm{k}), \ldots, \mathrm{u}_{j}\left(k+N_{C}-1\right)\right]}\left\{x^{m^{T}}\left(\mathrm{k}+N_{P}\right) P x^{m}\left(\mathrm{k}+N_{P}\right)+\right. \\
& \left.\left.\sum_{j=1}^{M} w_{j}\left\{\sum_{l=1}^{N_{P}}\left[x_{j}^{m^{T}}(\mathrm{~K}+l) \mathrm{Q}_{j} x_{j}^{m}(\mathrm{~K}+l)\right]+\sum_{l=0}^{N_{C}}-1 u_{j}^{m^{T}}(\mathrm{k}+l) \mathrm{R}_{j} u_{j}^{m}(\mathrm{k}+l)\right]\right\}\right\} \\
& \quad \text {.t. } \\
& \quad x_{j}\left(\mathrm{k}+q_{j}+1\right)=A_{j j} x_{j j}\left(k+q_{j}\right)+B_{j j} u_{j}\left(k+q_{j}\right)+B_{d_{j j}} d_{j}\left(k+q_{j}\right)+ \\
& \quad \sum_{i=1, \mathrm{i} \neq j}^{M} A_{j i} x_{i}\left(k+q_{j}\right)+B_{j i} u_{i}\left(k+q_{j}\right)+B_{d_{j i}} d_{i}\left(k+q_{j}\right), j \in\{1,2, \ldots, M\}
\end{aligned}
$$

Where $m \in Z_{+}$shows the number of iterations in the iterative algorithm $\left({ }^{Z_{+}}\right.$is the non-negative integer set) and $N_{P} \geq 1, N_{P} \in Z_{+}$ is prediction horizon and $N_{C} \geq 1, N_{C} \in Z_{+}$is control horizon. $\mathrm{Q}_{j} \succ 0, \mathrm{R}_{j} \succ o$ are weight symmetric matrices. Also value of $x_{j}(k)$ is considered as the initial state at time of $k$ for beginning of prediction. $\mathrm{X}^{\mathrm{T}}$ is the transpose of $\mathrm{X}$ and

$q_{j} \in\left\{0,1,2, \ldots, N_{P}-1\right\}, w_{j}>0, \sum_{j=1}^{M} w_{j}=1$. 
$A_{j j} \in R^{n_{j} \times n_{j}}, \quad B_{j j} \in R^{n_{j} \times m_{j}}, \quad B_{d_{j j}} \in R^{n_{j} \times s_{j}}$ are matrix coefficients of $\mathrm{j}$-th subsystem state space equation of and $d_{j}(\mathrm{k}) \in R^{s_{j}}$ is the bounded disturbance input at time $\mathbf{k}$ with upper bound given by (3).

$\left\|d_{j}\right\|_{2}^{2} \leq d_{\max }, d_{\max }>0, \forall j \in\{1,2, \ldots, M\}$

Also:

$\sum_{j=1}^{M} n_{j}=n, \sum_{j=1}^{M} m_{j}=m, \sum_{j=1}^{M} s_{j}=s$,

$w_{j} \succ 0, \sum_{j=1}^{M} w_{j}=1, \mathbf{x}=\left[\begin{array}{lll}\mathbf{x}_{1}^{T} & \ldots \mathbf{x}_{M}^{T}\end{array}\right]^{T}$

In addition, total feasible input sequences are characterized by $U_{j}$ set and the following equation:

$u_{j}^{m}\left(\mathrm{k}+q_{j}\right) \in U_{j}, q_{j}=0,1, \ldots, N_{C_{-}} 1$

Terminal penalty matrix $\mathrm{P}$ constrains terminal state $x\left(\mathrm{k}+N_{P}\right)$ to terminal set $s_{N_{P}}\left(r_{N_{P}}\right)$ and therefore, a priori guarantee for system stability is achieved [4] so that there are the following equations:

$$
\begin{aligned}
& s_{\mathrm{N}_{\mathrm{P}}}\left(r_{\mathrm{N}_{\mathrm{P}}}\right) \subseteq s_{\mathrm{q}}\left(r_{\mathrm{q}}\right) \quad, \quad r_{\mathrm{q}} \succ 0 \\
& s_{q}\left(r_{q}\right):=\left\{x(\mathrm{k}+q) \in R^{n} \mid\|x(\mathrm{k}+q)\|_{2}^{2} \leq r_{q}\right\}
\end{aligned}
$$

Where $s_{q}\left(r_{q}\right)$ is a closed set and a subset of $R^{n}$. Matrix $\mathrm{P}$ and matrix $\mathrm{K}$ are obtained by solving an unconstrained infinite horizon LQR problem as the following [10]:

$$
\begin{aligned}
& P=(A+B K) P(A+B K)+K R K+Q \\
& K=-(R+B P B) B P A
\end{aligned}
$$

If $K=0$ then (8) reduces to the Lyapunov equation $[4,7,10]$.

\section{APPLICATION IN POWER GRID}

Consider a power grid composed of 4 control zones for which tie-lines provide the connections between the zones. Each zone can contain several generators and several consumers that all of them can be modeled and lumped into a generator and a consumer.

Assuming that automatic generation control is limited to relatively small disturbances, the dynamics of each zone can be linearized, so the linear model and related specifications for case study will be offered later[4, 9, 11, 12].Then the standard model for zone $\mathrm{j}$ is obtained as follows:

$\frac{d \Delta P_{M E_{j}}}{d t}=\left(\Delta P_{V A j}-\Delta P_{M E j}\right) / \mathrm{t}_{C_{j}}$

$\frac{d \Delta \omega_{j}}{d t}=\left(\Delta P_{\mathrm{MEj}}-D_{j} \Delta \omega_{j}-\sum_{l=1}^{N E_{j}} \Delta P_{\mathrm{TIE}}^{k l}-\Delta P_{\mathrm{LD}_{j}}\right) / M_{j}$

$\frac{d \Delta P_{V A_{j}}}{d t}=\left(\Delta P_{R_{j}}-\Delta P_{V A_{j}}-\frac{\Delta \omega_{j}}{r_{j}^{F}}\right) / \mathrm{t}_{G_{j}}$

$\Delta P_{T I E}^{k l}=-\Delta P_{T I E}^{l k}$

$\frac{d \Delta P_{\mathrm{TIE}}^{k l}}{d t}=S_{k l}\left(\Delta \omega_{k}-\Delta \omega_{l}\right)$

In this type of modeling, the symbol $\Delta$ indicates deviation from steady state and $\mathrm{NE}_{j}$ represents the number of neighbors of subsystem $j$. This power grid is shown in Figure 1 and the list of related parameters is given in table 1 .

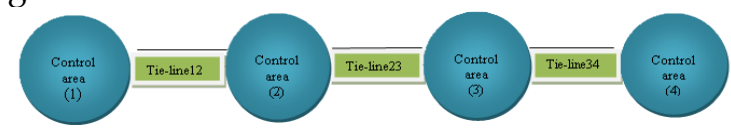

Fig. 1. A power grid consisting of 4 control zones

In each zone, local load variation causes variation in frequency. MPC controller by adjusting $P_{R}$ control input drives the frequency variations and the transmission power changes to zero. In this case, load demand change is considered as external disturbance.

\subsection{Simulated system specifications}


The sampling period is $\mathrm{T}=1 \mathrm{~s}$ and simulation time is 300 . We assume for discrete time instant $k \leq 30$ the grid is in the steady state and $\Delta \omega_{j}=\Delta P_{T I E}^{j h}=\Delta P_{L D_{j}}=0 . \quad$ For $30<k \leq 80$ the external disturbance in the value of $\Delta P_{L D_{2}}=-0.35, \Delta P_{L D_{3}}=+0.35$ enters the system as pulse shape in zones 2 and 3 . Also $\mathrm{N}_{\mathrm{P}}=60, \mathrm{~N}_{\mathrm{C}}=1$ and the maximum number of iterations of the algorithm at each time sample is $m_{\max }=8$. In addition, the range of control input changes is determined as follows:

$$
-0.15 \leq \Delta P_{R_{j}} \leq+0.15, j \in\{1,2,3,4\}
$$

Table 1. The parameters used in the simulation

\begin{tabular}{|c|}
\hline$\omega \mapsto$ angular frequency of system, $\delta \mapsto$ phase angle \\
\hline $\mathrm{P}_{\mathrm{ME}} \mapsto$ mechanical power, $\mathrm{P}_{\mathrm{VA}} \mapsto$ steam valve position \\
\hline $\mathrm{P}_{\mathrm{LD}} \mapsto$ nonfrequency sensitive load, $\mathrm{P}_{\mathrm{R}} \mapsto$ load refrence setpoint \\
\hline $\mathrm{P}_{\mathrm{TIE}}^{\mathrm{kl}} \mapsto$ tie line power flow between areas $\mathrm{k} \& \mathrm{l}$ \\
\hline $\mathrm{M} \mapsto$ angular momentum, $\mathrm{t}_{\mathrm{G}} \mapsto$ governor time cons $\tan \mathrm{t}$ \\
\hline $\mathrm{D} \mapsto(\%$ change in load $) /(\%$ change in frequency $)$ \\
\hline $\mathrm{r}^{\mathrm{F}} \mapsto(\%$ change in frequency $) /(\%$ change in unit output $)$ \\
\hline $\mathrm{S}_{\mathrm{kl}} \mapsto$ tie line stiffness coefficient (between areas $\mathrm{k} \& 1$ ) \\
\hline $\mathrm{t}_{\mathrm{C}} \mapsto$ charching time cons tan $\mathrm{t}$ \\
\hline
\end{tabular}

State variables are defined as follows:

Zone 1: $\Delta P_{V A 1}, \Delta P_{M E 1}, \Delta \omega_{1}$

Zone 2: $\Delta \delta_{12}, \Delta P_{V A 2}, \Delta P_{M E 2}, \Delta \omega_{2}$

Zone 3: $\Delta \delta_{23}, \Delta P_{V A 3}, \Delta P_{M E 3}, \Delta \omega_{3}$

Zone $4: \Delta \delta_{34}, \Delta P_{V A 4}, \Delta P_{M E 4}, \Delta \omega_{4}$

\subsection{Automatic generation control by Classical method}

The traditional AGC (automatic generation control) method uses the PI controller. In this regard, the following controller is used for zone $\mathrm{j}[4]$ :

$\Delta P_{R_{j}}=-R_{j} \int_{t^{\prime}=-\infty}^{t}\left(E_{j} \Delta \omega_{j}+\sum_{h=1}^{N E_{j}} \Delta P_{T I E}^{j h}\right) d t^{\prime}$

$R_{j}, E_{j}$ are PI controller tuning parameters. Error is calculated for each zone by the following equation [13]:

$\operatorname{ERROR}_{j}(\mathrm{k})=b_{j} \times \Delta \omega_{j}(k)+\sum_{i=1, i \neq j}^{N E_{j}} \Delta P_{T I E}^{j i}(k)$

$b_{j}$ is relative weighting coefficient of error outputs.

Total error is determined by the following equation:

TOTAL_ERROR $(k)=\Sigma_{j=1}^{4} \operatorname{ERROR}_{j}(\mathrm{k})$

In order to examine and compare overall performance of the system, the outputs of zone 1 and zone 3 and the total error of system are shown in Figure 2. Zone 1 is without disturbance input but zone 3 includes load pulse changes as disturbance input. Total error is collective combination of existing errors including frequency variations and transmission power variations between the zones. 


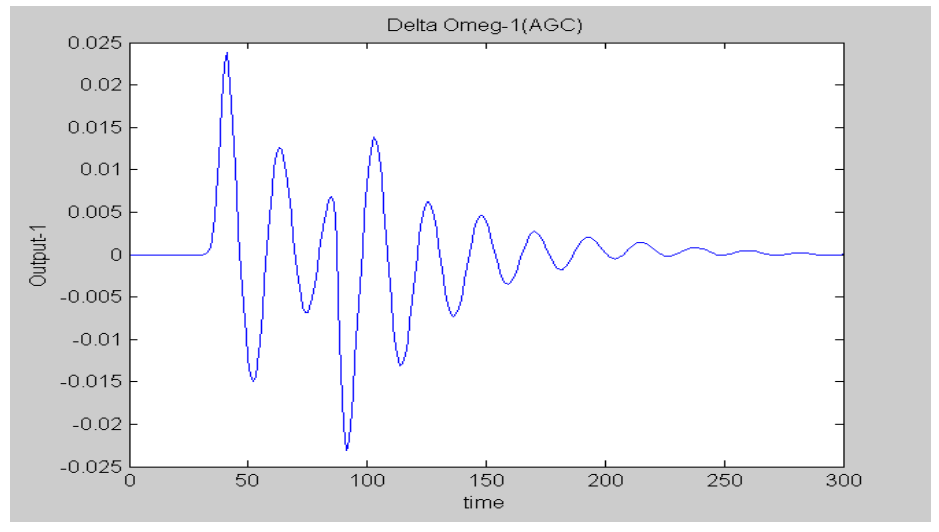

(a)

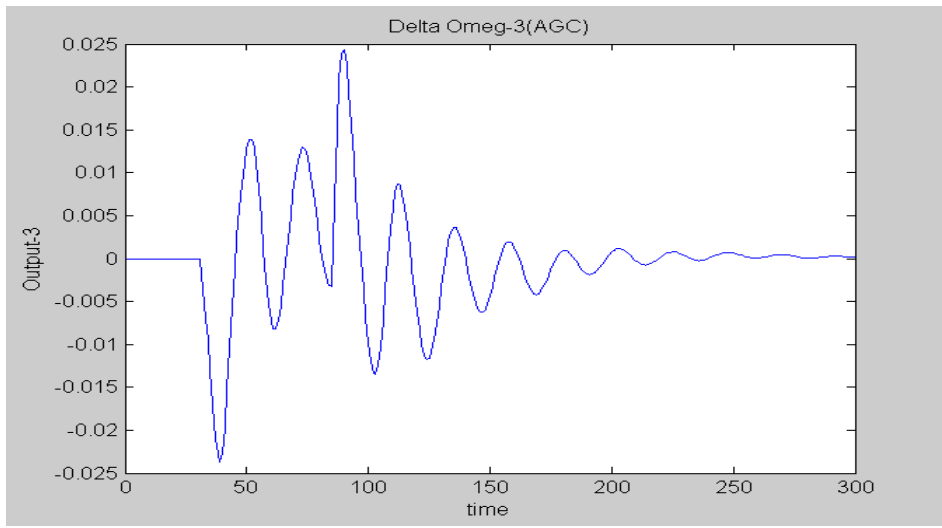

(b)

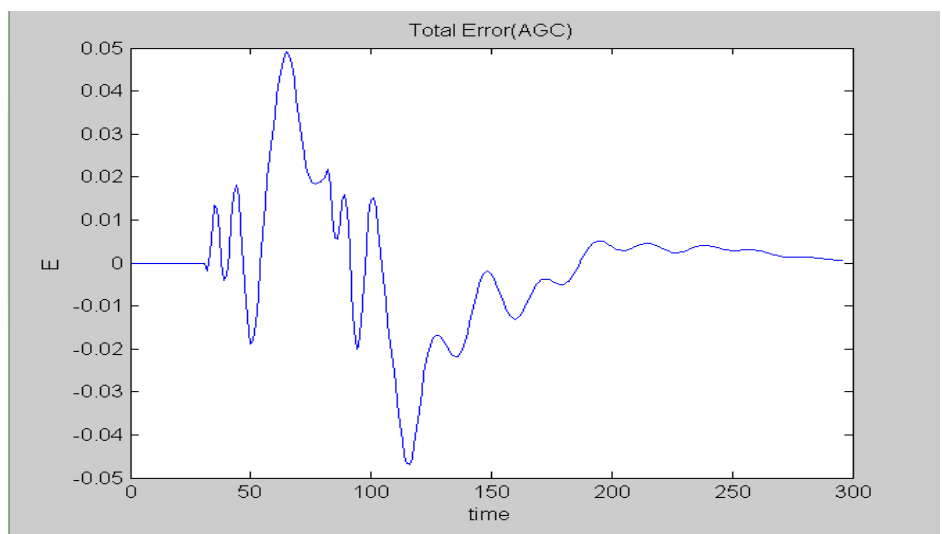

( c )

Fig. 2. The results related to classical AGC : a) frequency changes in zone $1, \boldsymbol{b}$ ) frequency changes in zone $3, \mathrm{c}$ ) total error

4.3. Generation control by the cooperative distributed predictive method

In Figure 3, the same as previous section, to evaluate overall performance of control system, curves of frequency changes for zone 1 (without local load changes) and zone 3 (with local load pulse changes as external disturbance input) and curve of total error have been shown. Figure 3 clearly demonstrates the error caused by load disturbance that includes the deviations in 
frequency and transmission power, can be

performance. gradually decreased by good control

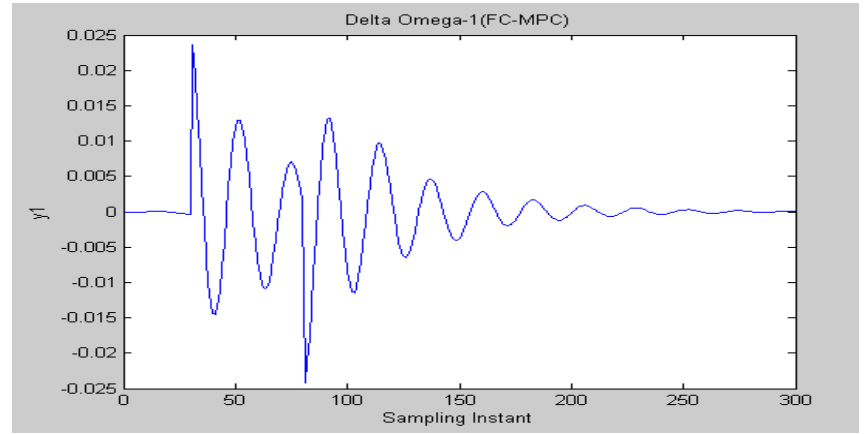

(a)

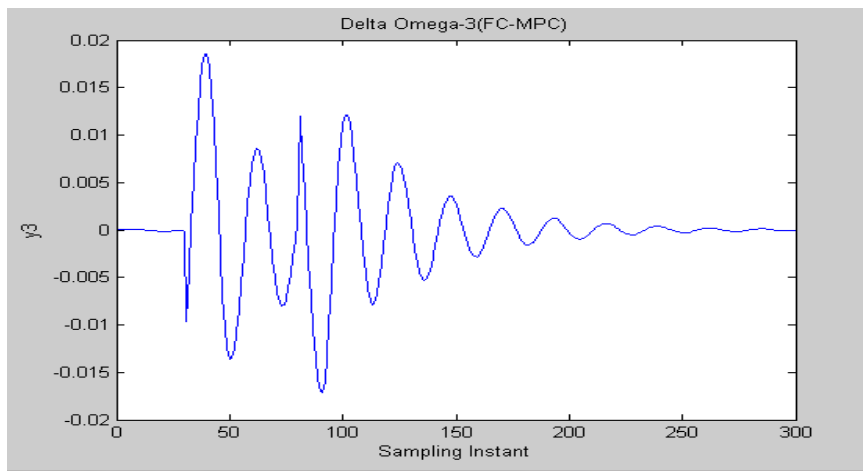

(b)

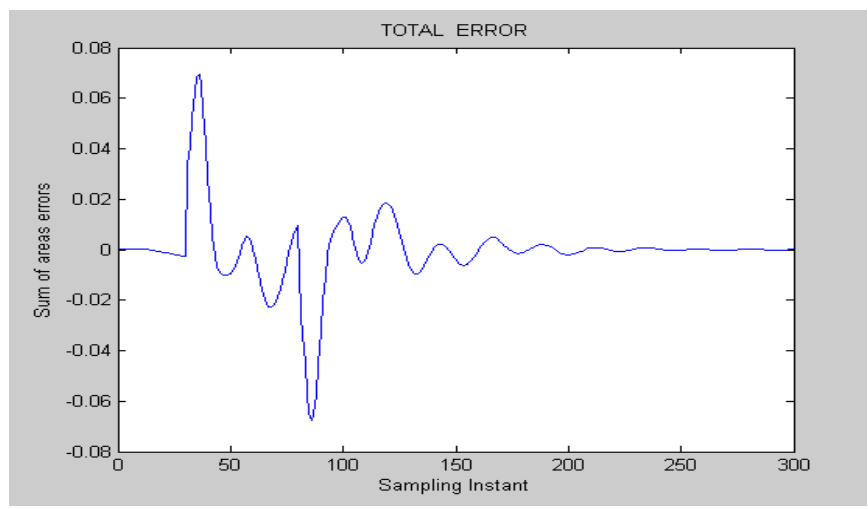

( c )

Fig. 3. The results related to FC-MPC : a) frequency changes in zone $1, \boldsymbol{b}$ ) frequency changes in zone 3, c) total error

\section{DISCUSSION OF SIMULATION RESULTS}

Here, settling time is the time required for a signal to settle within an error band of $\% 5$ around the steady-state value. Norm- 1 of error indicates sum of absolute value of signal in all of simulation time instants and terminal value points the value of signal in the last simulation time instant. These values and their differences in terms of percentage are listed in Table 2, for total error signals related to AGC and FCMPC methods. This 
table shows that specifications of FCMPC are better than AGC method.

Table 2. Comparison between specifications of error signals

\begin{tabular}{|l|c|c|c|}
\hline signal specification & Norm-1 & Settling time & $\begin{array}{l}\text { Terminal value } \\
\text { (absolute value) }\end{array}$ \\
\hline Total Error(AGC) & 2.7810 & $\mathbf{2 6 2 . 9 4 0 6}$ & $6.2646 \mathrm{e}-4$ \\
\hline Total Error(FCMPC) & 2.0108 & $\mathbf{1 6 9 . 4 4 7 4}$ & $8.4858 \mathrm{e}-5$ \\
\hline Difference (\%) & $\mathbf{3 8 . 3 0}$ & $\mathbf{5 5 . 1 8}$ & $\mathbf{6 3 8 . 2 5}$ \\
\hline
\end{tabular}

\section{CONCLUSION}

The results obtained in Sections 4.2 and 4.3 indicate that frequency control in given grid is done properly by cooperative distributed predictive control method, even after successive load changes and with stricter constraints. Finally, after a specified time of load disturbance, the grid returns to steady state by decaying frequency deviations and total error.

The overall goals of system can be achieved with cooperative distributed MPC structure regarding to interaction between controllers of the different zones. Also it facilitates the calculations in comparison with centralized control structure. However, by increasing communication, the system complexity is increased somewhat more than the decentralized type and computational speed is decreased, but it should be noted that AGC is a relatively slow but precise process [14] and increasing cooperation between control zones in the system may improve its performance. Of course in the new researches to improve this structure, techniques are still being studied and offered.

On the other hand, one of the most important issues in power grids is preservation quality of electric power, especially after making them smart in the future. So it is essential to improve the adjustment of frequency within an admissible range. It would be expected that with activity of power quality market , this issue will also have specific and measurable economic value. Moreover, with the expansion of the smart grids, it seems essential that the modern control methods with advanced specifications should be used in place of traditional methods. In this regard, predictive control with appropriate architecture can be used as a efficient control strategy that offers a kind of foresight in design and implementation of a control system.

\section{Acknowledgement}

We are grateful of Kerman Regional Electric Company (KREC) cooperation.

\section{REFERENCES}

Dragoslav D. siljak (1991). "decentralized control of complex systems" Academic press limited.

N. R. Sandell, Jr., P. Varaiya, M. Athans, and M. Safonov,(1978) "Survey of decentralized control methods for large scale systems" ,IEEE Trans. Autom. Control, vol. 23, no. 2, pp. 108-128,

Dragoslav D. siljak, (1978)“ Large-scale Dynamic Systems: Stability and Structure" . North-Holland.

Ralph M. Hermans, Andrej Jokic', Mircea Lazar, Alessandro Alessio, Paul P.J. van den Bosch,Ian A.Hiskensand Alberto Bemporad ,(2012) "Assessment of non- 
centralized model predictive control techniques for electrical power networks" , International Journal of Control ,Vol. 85, No. 8, 1162-1177.

Liuping Wang ,2009) “Model Predictive Control System Design and implementation using MATLAB", , Springer.

Cui, H., \& Jacobsen, E. (2002), "Performance limitations in decentralized control", Journal of Process Control, 12,485-494.

Alessio, A., and Bemporad, A. ,(2007), "Decentralized Model Predictive Control of Constrained Linear Systems", in Proceedings European Control Conference, Kos,Greece, pp. 2813-2818.

Venkat, A.N. ,(2006), “Distributed Model Predictive Control : Theory and Applications" , PhD Thesis, University of Wisconsin-Madison, Madison, WI, USA.

Venkat, A.N., Hiskens, I.A., Rawlings, J.B., and Wright, S.J. ,(2008), “Distributed MPC Strategies with Application to Power System Automatic Generation Control" , IEEE Transactions on Control Systems Technology, 16, 1192-12

Mayne, D.Q., Rawlings, J.B., Rao, C.V., and Scokaert,P.O.M. ,(2000), "Constrained Model Predictive Control : Stability and Optimality", Automatica, 36, 789-814.

Kundur, P.,( 1994), "Power System Stability and Control" "New York, NY, USA: McGraw-Hill.

A. J. Wood and B. F. wollenberg ,(1996), "Power Generation Operation and Control", New York: Wiley.

Miaomiao Ma, Hong Chen, Xiangjie Liu , Frank Allgöwer , (2014), “Distributed model predictive load frequency control of multi-area interconnected power system" , Electrical Power and Energy Systems 62 , 289-298.

Jaleeli, N., VanSlyck, L., Ewart, D., Fink, L., and Hoffmann, A., (1992), "Understanding Automatic Generation Control" , IEEE Transactions on Power Systems, Vol. 7, No. 3, 1106-1122. 